\title{
EFEITO DA APLICAÇÃO SEQÜENCIAL DE GLYPHOSATE NO CONTROLE DE Commelina benghalensis E Commelina diffusa NA CULTURA DO CAFEEIRO
}

\author{
Ricardo C. Werlang ${ }^{1}$, Lino Roberto Ferreira ${ }^{2}$ e Antônio Alberto da Silva ${ }^{3}$
}

${ }^{1}$ Eng ${ }^{\circ}$. Agrônomo, Mestrando. UFV/Dep. de Fitotecnia. Av. P. H. Rolfs, s/n. Viçosa, MG 36571-000 rwerlang@hotmail.com ${ }^{2}$ Professor. UFV/Departamanto de Fitotecnia. Iroberto@mail.ufv.br

${ }^{3}$ Professor. UFV/Departamento de Fitotecnia. aasilva@mail.ufv.br

\section{RESUMO}

A fim de estabelecer estratégias que reduzam a tolerância de Commelina benghalensis e C. diffusa ao glyphosate, foram realizados dois experimentos lavoura de café. Foi avaliado o efeito da aplicação seqüencial de doses crescentes de glyphosate, comparando com as formulações de glyphosate SA (sal isopropilamina) e WG (sal amônio) no controle destas espécies e a toxicidade causada à cultura. Não foi observada diferença significativa entre as formulações glyphosate SA (2160 g/ha e.a.) e WG ( $2160 \mathrm{~g} /$ ha e.a.), no controle de $C$. diffusa e C. benghalensis. A aplicação seqüencial de glyphosate WG, nas doses de 1440 e $1080 \mathrm{~g} /$ ha e.a., com a segunda aplicação 21 dias após a primeira, demonstrou controle eficiente de $C$. diffusa e C. benghalensis, até os 100 dias após a aplicação (DAA). A mistura no tanque glyphosate SA + 2,4-D na dose de 1080/1005 $\mathrm{g} /$ ha e.a. e a mistura diuron/paraquat na dose de 300/600 g/ha, proporcionaram injúria acentuada ao cafeeiro até os 60 DAA. No entanto, glyphosate SA e glyphosate WG em aplicação seqüencial ou em única aplicação, nas doses estudadas, ocasionou injúria acentuada ao cafeeiro até os $30 \mathrm{DAA}$, passando para injúrias leves nas avaliações seguintes.

Palavras-chave: 2,4-D, fitotoxicidade, formulações de glyphosate, mistura no tanque, paraquat.

\section{ABSTRACT \\ Effect of the sequential application of glyphosate in Commelina benghalensis and Commelina diffusa control in coffee crop}

Different strategies to reduce $C$. benghalensis and $C$. diffus a tolerance to glyphosate were studied in two field experiments in coffee. The effect of glyphosate sequential application at increasing doses and comparing the glyphosate formulations, glyphosate SA (isopropilamine salt) and glyphosate WG (ammonium salt) in the control of these species and the respective phytotoxicity was evaluated. No significant difference were found among formulations of glyphosate SA (2160 g/ha a.e.) and glyphosate WG (2160 g/ha a.e.), in C. diffusa and C. benghalensis control. The sequential application of glyphosate WG at 1440 and $1080 \mathrm{~g} /$ ha a.e., with the second application 21 days after the first, was efficient for $C$. diffusa and $C$. benghalensis control, from 30 to 100 days after application (DAA). The tank mixture glyphosate SA+2,4-D, at $1080+1005 \mathrm{~g} / \mathrm{ha}$ a.e., and the mixture diuron/paraquat at 300/600 g/ha resulted in accentuated phytotoxicity until 60 DAA. However, glyphosate SA and glyphosate WG in sequential application or in unique application, in the studied doses, caused accentuated phytotoxicity until 30 DAA, turning to light phytotoxicity in the following evaluations.

Key words: 2,4-D, glyphosate formulations, paraquat, phytotoxicity, tank mixture. 


\section{INTRODUÇÃO}

A cultura cafeeira possui grande importância, em virtude da geração de emprego e maior renda nas propriedades rurais, além de divisas na exportação e de impostos para o País.

Segundo Lorenzi (2000), as plantas daninhas afetam diretamente a produção das culturas, pela competição por luz, umidade e nutrientes minerais, ou indiretamente, pelo aumento de inóculo ou pela manutenção de organismos patogênicos. As plantas daninhas, além da competição pela umidade do solo e por nutrientes essenciais, interferem em práticas culturais, como controle de pragas, fertilização e colheita.

Segundo Blanco et al. (1982), o modo correto de se interferir na competição seria neutralizá-la nas épocas adequadas, ou seja, nos períodos em que as plantas daninhas provocam concorrência e prejudicam a produção. A competição é diferenciada conforme a época do ano. Conforme esses autores, considerando o balanço hídrico da região de São Paulo, com épocas de chuva e seca bem definidas ao longo do ano, quando a competição é eliminada no período de maior precipitação pluvial (outubro a abril), que engloba as fases de florescimento e frutificação do cafeeiro, a produção não é comprometida. A não eliminação da competição no mesmo período ocasionou reduções na produção, de até $77,2 \%$. É possível que o aumento da produção de café se deva ao aumento do número de dias sem competição, iniciando-se a limpeza em outubro.

A família Commelinaceae apresenta mais de 40 gêneros; destes, o Commelina é o mais importante, de ampla distribuição no Brasil, sendo conhecido pelo nome comum de trapoeraba (Kissmann, 1997). Mesmo existindo vários representantes deste gênero no Brasil (Barreto, 1997), apenas as espécies Commelina benghalensis e C. diffusa são infestantes em Minas Gerais.

Dentre os vários métodos de controle, o químico tem se destacado pela sua aplicabilidade em períodos chuvosos, permitindo ainda a persistência da cobertura morta por maior tempo sobre o solo, reduzindo a produção e proporcionando controle por tempo mais prolongado (Njoroge, 1994), além de ser de baixo custo e de rápida operação, em comparação ao controle mecânico.

A importância dessas duas espécies, no manejo químico de plantas daninhas, refere-se à sua tolerância aos herbicidas mais utilizados: glyphosate e sulfosate (Vargas et al., 1999). Estes são de uso generalizado, por apresentarem controle eficiente sobre a maioria das espécies de plantas daninhas.

O glyphosate pertence ao grupo dos inibidores da síntese de aminoácidos e contém o N-(fosfonometil) glicina como ingrediente ativo (Rodrigues \& Almeida, 1998). Atualmente estão disponíveis no mercado vários herbicidas com este ingrediente ativo, porém com sal isopropanolamina, potássico ou amônio, na constituição do produto final (Hartzler, 2001).
O glyphosate é um produto sistêmico e não-seletivo, altamente solúvel em água e seu mecanismo de ação baseia-se na interrupção da rota do ácido chiquímico, responsável pela produção dos aminoácidos aromáticos fenilalanina, tirosina e triptofano, que são essenciais para a síntese de proteínas e divisão celular nas regiões meristemáticas da planta (Hess, 1994).

Na maioria das plantas, o glyphosate é rapidamente translocado das folhas tratadas para os drenos metabólicos, especialmente tecidos meristemáticos e de armazenagem (Bromilow et al., 1990), sendo, por isso, excelente herbicida para o controle de plantas daninhas perenes. Outra vantagem do glyphosate é o fato dele apresentar baixa toxicidade à vida aquática e aos mamíferos, considerando que esses aminoácidos não são sintetizados por esses organismos, sendo ainda rapidamente inativado no solo (Rodrigues \& Almeida, 1998).

Uma opção no controle de trapoeraba, com glyphosate, é a utilização de aplicações seqüenciais. Dessa forma, a primeira dose do herbicida predispõe as plantas daninhas a maior sensibilidade quanto ao efeito do herbicida na segunda aplicação. Essas estratégias que influenciam a tolerância da trapoeraba ao glyphosate são de extrema importância ao conceito de manejo integrado de plantas daninhas, devido às excelentes características do produto e ao difícil controle destas espécies na cultura cafeeira.

Os objetivos deste trabalho foram verificar o efeito da aplicação seqüencial de doses crescentes de glyphosate e comparar as formulações de glyphosate SA (sal isopropamolamina) e glyphosate WG (sal amônio) no controle de $C$. benghalensis e $C$. diffusa na cultura do cafeeiro e a respectiva toxicidade causada à cultura.

\section{MATERIAL E MÉTODOS}

Foram realizados dois experimentos no período de março a junho de 2001. Um destes foi realizado na fazenda Jatobá, em área comercial de café, variedade Catuaí Vermelho, com dois anos de idade e implantada no espaçamento de $3,0 \times 1,0 \mathrm{~m}$, possuindo área infestada com $C$. benghalensis. $\mathrm{O}$ outro experimento foi realizado em área infestada com $C$. diffusa, pertencente à Universidade Federal de Viçosa, em lavoura de café, variedade Catuaí Vermelho, com quatro anos de idade e implantada no espaçamento de 3,0 x 1,0 m. As propriedades são localizadas no município de Viçosa, $\mathrm{MG}$, situada geograficamente a $20^{\circ} 50^{\prime} 30^{\prime \prime}$ de latitude sul e a $42^{\circ}$ $48^{\prime} \mathrm{e} 30^{\prime \prime}$ de longitude oeste, com altitude de $716 \mathrm{~m}$.

No momento da aplicação, em ambos os experimentos a área apresentava aproximadamente $90 \%$ de cobertura com as espécies $C$. benghalensis e $C$. diffusa, respectivamente para cada experimento. As plantas daninhas adultas estavam no início da fase reprodutiva e apresentavam ótimo desenvolvimento. Os tratamentos foram constituídos pela aplicação seqüencial de doses crescentes de glyphosate, com a 
segunda aplicação 21 dias após a primeira, como pode ser observado na Tabela 1.

Foi utilizado o delineamento experimental de blocos ao acaso, com 10 tratamentos e quatro repetições. Cada parcela possuía $6,0 \mathrm{~m}$ de comprimento e 3,0 $\mathrm{m}$ de largura.

As aplicações dos tratamentos foram feitas com um pulverizador costal pressurizado com $\mathrm{CO}_{2}$, mantendo-se a pressão constante em $3,0 \mathrm{kgf} / \mathrm{cm}^{2}$. Foi aplicado o equivalente a $200 \mathrm{~L} /$ ha de calda, usando-se uma barra com quatro bicos TT-110.02. No momento da primeira aplicação (15/03/01, às 10 horas) a velocidade do vento era inferior a $5 \mathrm{~km} / \mathrm{h}$, com temperatura de $27^{\circ} \mathrm{C}$ e umidade relativa do ar (UR) de $72 \%$. Na segunda aplicação (05/04/01, às 9 h30) a velocidade do vento era inferior a $5 \mathrm{~km} / \mathrm{h}$, com temperatura de $28^{\circ} \mathrm{C}$ e UR de $73 \%$.

A eficácia de controle das espécies avaliadas foi verificada aos $15,30,45,60$ e 100 dias após a primeira aplicação (DAA), com base nos sintomas de toxicidade, utilizando-se a escala de Frans (1972), em que 0\% corresponde à ausência de sintomas visíveis e $100 \%$ à morte das plantas. As avaliações de fitotoxicidade foram realizadas aos 15, 30, 45 e 60 dias após a primeira aplicação (DAA), obedecendo à escala da EWRC, observando-se as folhas baixeiras dos ramos plagiotrópicos das plantas do cafeeiro na parcela.

Os dados obtidos nas avaliações de controle e toxicidade foram submetidos à análise de variância e as médias comparadas pelo teste de Tukey a 5\% de probabilidade.

A área experimental recebeu todos os tratos culturais necessários ao bom desenvolvimento e produtividade da cultura. Os dados meteorológicos obtidos durante a execução do experimento são apresentados na Figura 1.

\section{RESULTADOS E DISCUSSÃO}

O quadrado médio obtido através da análise de variância para as percentagens de controle de Commelina diffusa e Commelina benghalensis e a toxicidade ocasionada ao cafeeiro nas avaliações realizadas aos 15, 30, 42, 60 e 100 DAA podem ser observados na Tabela 2.

A mistura diuron/paraquat $(300 / 600 \mathrm{~g} / \mathrm{ha})$ demonstrou controle excelente de $C$. diffusa (93\%) apenas aos 15 DAA, apresentando redução do controle nas avaliações posteriores, devido ao restabelecimento da planta daninha. Já $C$. benghalensis apresentou controle moderado a insuficiente (75\%) aos 15 DAA, demonstrando redução do controle nas avaliações seguintes. A ocorrência de precipitações neste período proporcionou o rápido restabelecimento das espécies daninhas (Figura 1).

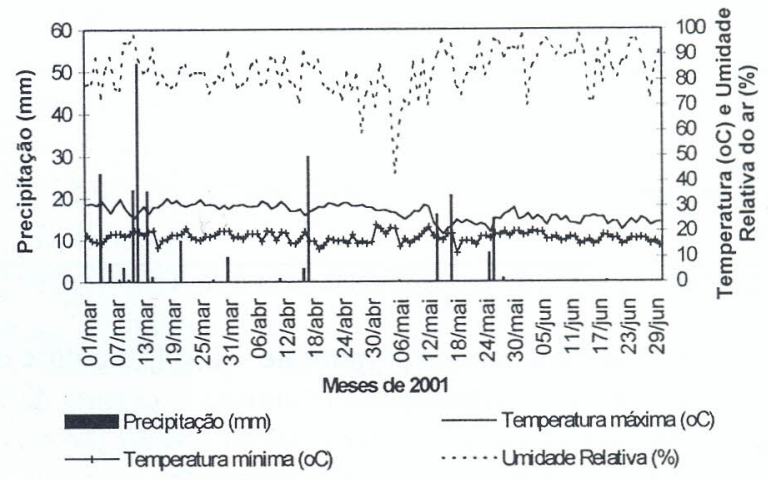

Figura 1. Dados meteorológicos observados no período de execução do experimento. Viçosa, MG, 2001.

Tabela 1. Tratamentos herbicidas avaliados nos experimentos de controle de Commelina benghalensis e $C$. diffusa na cultura cafeeira, com aplicações feitas em 15/03/01 (primeira aplicação) e em 05/04/01 (segunda aplicação). Viçosa, MG, 2001.

\begin{tabular}{|c|c|c|c|c|c|}
\hline \multirow{2}{*}{\multicolumn{2}{|c|}{ Herbicida }} & \multicolumn{4}{|c|}{ Dose } \\
\hline & & Primei & plicação & Segun & cação \\
\hline Nome comum & Produto comercial & $\begin{array}{c}\text { g/ha } \\
\text { (e.a. ou i.a.) }\end{array}$ & $\begin{array}{c}\mathrm{kg} / \mathrm{ha} \text { ou L/ha } \\
\text { (p.c.) }\end{array}$ & $\begin{array}{l}\text { g/ha } \\
\text { (e.a.) }\end{array}$ & $\begin{array}{l}\mathrm{kg} / \mathrm{ha} \\
\text { (pc) }\end{array}$ \\
\hline Glyphosate WG & Roundup Multiação $^{1}$ & 720 & 1,0 & 1.080 & 1,5 \\
\hline Glyphosate WG & Roundup Multiação & 1080 & 1,5 & 1.080 & 1,5 \\
\hline Glyphosate WG & Roundup Multiação & 1440 & 2,0 & 1.080 & 1,5 \\
\hline Glyphosate WG & Roundup Multiação & 1800 & 2,5 & 1.080 & 1,5 \\
\hline Glyphosate WG & Roundup Multiação & 1080 & 1,5 & -- & -- \\
\hline Glyphosate WG & Roundup Multiação & 2160 & 3,0 & --- & --- \\
\hline Glyphosate SA & Roundup Original ${ }^{2}$ & 2160 & 6,0 & --- & --- \\
\hline Glyphosate SA + 2,4-D & Roundup Original + DMA $806^{3}$ & $1080+1005$ & $3,0+1,5$ & -- & --- \\
\hline Diuron/paraquat & Gramocil $^{4}$ & $300 / 600$ & 3,0 & -- & --- \\
\hline
\end{tabular}

'Formulação comercial contendo 72\% de glyphosate sal amônio; ${ }^{2 /}$ Formulação comercial contendo 36\% de glyphosate sal isopropilamina; ${ }^{3}$ Formulação comercial contendo $67 \%$ de 2,4-D amina; ${ }^{4 /}$ Formulação comercial contendo $10 \%$ de diuron $/ 20 \%$ de paraquat. 
Ricardo C. Werlang et al.

Tabela 2. Resumo das análises de variância dos dados referentes às percentagens de controle de Commelina diffusa e Commelina benghalensis e da toxicidade ocasionada ao cafeeiro pelos tratamentos nas diferentes épocas de avaliação. Viçosa, MG, 2001.

\begin{tabular}{|c|c|c|c|c|c|}
\hline \multirow{2}{*}{ Fonte de variação } & \multicolumn{5}{|c|}{ Quadrado médio } \\
\hline & $15 \mathrm{DAA}$ & $30 \mathrm{DAA}$ & 42 DAA & $60 \mathrm{DAA}$ & $100 \mathrm{DAA}$ \\
\hline \multicolumn{6}{|c|}{ Commelina benghalensis } \\
\hline Herbicidas & $1994,966 * *$ & $807,465 * *$ & $1963,73 * *$ & $3228,25 * *$ & $3304,84 * *$ \\
\hline $\mathrm{CV}$ & 23,7 & 11,1 & 8,3 & 10,6 & 8,0 \\
\hline \multicolumn{6}{|c|}{ Commelina diffusa } \\
\hline Herbicidas & $1883,063 * *$ & $490,527 * *$ & $1530,986 * *$ & $1917,237^{* *}$ & $986,63 * *$ \\
\hline $\mathrm{CV}$ & 12,9 & 9,4 & 8,6 & 8,3 & 10,3 \\
\hline \multicolumn{6}{|c|}{ Toxicidade ocasionada ao cafeeiro } \\
\hline Herbicidas & $404,687 * *$ & $151,736 * *$ & $296,562 * *$ & $307,749 * *$ & --- \\
\hline $\mathrm{CV}$ & 14,7 & 26,2 & 26,9 & 24,3 & --- \\
\hline
\end{tabular}

** Significativo a $1 \%$ de probabilidade pelo teste $\mathrm{F}$.

A mistura em tanque glyphosate $+2,4-\mathrm{D}$, na dose de $1080+1005 \mathrm{~g} /$ ha e.a., demonstrou controle excelente de $C$. diffusa $(>99 \%)$ e $C$. benghalensis ( $>93 \%$ ) dos 30 DAA aos 100 DAA; no entanto, aos 15 DAA apresentou controle insuficiente para $C$. diffusa (85\%) e C. benghalensis (78\%).

Glyphosate WG, com aplicação única, nas doses de 1080 e $2160 \mathrm{~g} /$ ha e.a., não foi eficaz no controle das espécies avaliadas $(<90 \%)$, diferindo dos resultados obtidos por Santos et al. (2001), em que o glyphosate controlou eficazmente C. benghalensis a partir de $720 \mathrm{~g} / \mathrm{ha}$ e.a. Contudo, para $C$. diffusa, um controle excelente por mais de 60 dias só foi obtido a partir de $2880 \mathrm{~g} /$ ha e.a. Essas diferenças possivelmente estejam relacionadas às condições de desenvolvimento das plantas daninhas, uma vez que os autores cultivaram as espécies em bandejas plásticas e, no presente trabalho, as avaliações foram realizadas no campo, sendo observado condições adequadas ao desenvolvimento das espécies (Figura 1). No entanto, Ramos \& Durigan (1996) relatam a seleção de espécies de trapoeraba pelo uso generalizado de glyphosate na cultura de citros, que passou a ter grande importância na referida cultura, devido à dificuldade de controle. A ineficiência do glyphosate no controle de trapoeraba é também relatada por outros autores (Durigan et al., 1988; Santos et al., 2000).

Não ocorreu diferença significativa entre as formulações glyphosate SA (2160 g/ha e.a.) e glyphosate WG (2160 $\mathrm{g} / \mathrm{ha}$ e.a.), em única aplicação, no controle das espécies e nas épocas avaliadas.

Observa-se, que a aplicação seqüencial de glyphosate WG na dose de 1440 e $1080 \mathrm{~g} /$ ha e.a. demonstrou controle excelente de $C$. diffusa (>91\%) aos 42 e 60 DAA, possuindo controle moderado (>84\%) aos 30 e 100 DAA (Tabela 3). Por outro lado, na dose de 1800 e $1080 \mathrm{~g} /$ ha e.a. houve controle excelente dos $30 \mathrm{DAA}$ aos $60 \mathrm{DAA}$, apresentando controle moderado aos 100 DAA. Para $C$. benghalensis, a aplicação seqüencial de glyphosate WG na dose de 1440 e 1080 $\mathrm{g} / \mathrm{ha}$ e.a. demonstrou controle excelente da espécie a partir dos 42 DAA, apresentando controle moderado aos 30 DAA (Tabela 4).

Os tratamentos com glyphosate WG em aplicação seqüencial não diferiram do tratamento-padrão glyphosate $\mathrm{SA}+2,4-\mathrm{D}$ na dose de $1080+1005 \mathrm{~g} /$ ha e.a., no controle de C. diffusa dos 30 aos 100 DAA. No entanto, no controle de C. benghalensis, somente as aplicações seqüenciais de glyphosate SA nas duas maiores doses (1440 e 1080, e 1800 e $1080 \mathrm{~g} / \mathrm{ha}$ e.a.) não diferiram significativamente do tratamento glyphosate $\mathrm{SA}+2,4-\mathrm{D}$ nas mesmas épocas.

Todas as formulações testadas caso atinjam as folhas do café causam injúria. No entanto os tratamentos que proporcionaram maior injúria foram a mistura no tanque glyphosate SA + 2,4-D na dose de $1080+1005$ g/ha e.a. e a mistura comercial diuron/paraquat na dose de $300 / 600 \mathrm{~g} / \mathrm{ha}$ (Tabela 5).

A mistura no tanque de glyphosate $+2,4-\mathrm{D}$ requer tecnologia de aplicação adequada e maior cuidado, a fim de garantir o controle das plantas daninhas e ocasionar a menor injúria possível ao cafeeiro. A maioria desses problemas é causada pelo 2,4-D; este herbicida pode ser translocado no solo, penetrando pelo sistema radicular da cultura, e também ser carregado pelo vento, atingindo as folhas do café, devido às suas características físicas e químicas. 
Efeito da aplicação sequencial de glyphosate na cultura do cafeeiro

Tabela 3. Controle de Commelina diffusa aos 15, 30, 42, 60 e 100 dias após aplicação (DAA) na cultura cafeeira. Viçosa, MG, 2001.

\begin{tabular}{|c|c|c|c|c|c|c|c|}
\hline \multirow[b]{2}{*}{ Tratamento } & \multicolumn{2}{|c|}{ Dose (g/ha) } & \multicolumn{5}{|c|}{ Controle $^{(\%)}$} \\
\hline & $1^{\mathrm{a}}$ Aplicação & $2^{\mathrm{a}}$ Aplicação & 15 DAA & 30 DAA & 42 DAA & 60 DAA & 100 DAA \\
\hline Glyphosate WG & 720 & 1080 & $33 \mathrm{~b}$ & 81 abcde & $94 \mathrm{ab}$ & $88 \mathrm{ab}$ & $74 \mathrm{ab}$ \\
\hline Glyphosate WG & 1080 & 1080 & $35 \mathrm{~b}$ & 85 abcd & $95 \mathrm{ab}$ & $89 \mathrm{ab}$ & $73 \mathrm{ab}$ \\
\hline Glyphosate WG & 1440 & 1080 & $39 \mathrm{~b}$ & $88 \mathrm{abc}$ & $96 \mathrm{ab}$ & $91 \mathrm{ab}$ & $84 \mathrm{ab}$ \\
\hline Glyphosate WG & 1800 & 1080 & $46 \mathrm{~b}$ & $90 \mathrm{ab}$ & $97 \mathrm{ab}$ & $94 \mathrm{a}$ & $84 \mathrm{ab}$ \\
\hline Glyphosate WG & 1080 & - & $45 \mathrm{~b}$ & $66 \mathrm{e}$ & $81 \mathrm{~b}$ & $78 \mathrm{~b}$ & $68 \mathrm{bc}$ \\
\hline Glyphosate WG & 2160 & - & $48 \mathrm{~b}$ & 79 bcde & $88 \mathrm{ab}$ & $84 \mathrm{ab}$ & $71 \mathrm{~b}$ \\
\hline Glyphosate SA & 2160 & - & $45 \mathrm{~b}$ & 68 de & $83 a b$ & $84 \mathrm{ab}$ & $81 \mathrm{ab}$ \\
\hline Glyphosate SA + 2,4-D & $1080+1005$ & - & $85 \mathrm{a}$ & $100 \mathrm{a}$ & $100 \mathrm{a}$ & $99 \mathrm{a}$ & $99 \mathrm{a}$ \\
\hline Diuron/paraquat & $300+600$ & - & $93 \mathrm{a}$ & 71 cde & $36 \mathrm{c}$ & $25 \mathrm{c}$ & $41 \mathrm{c}$ \\
\hline Testemunha sem capina & - & - & 0 & 0 & 0 & 0 & 0 \\
\hline
\end{tabular}

${ }^{(1)}$ Médias seguidas pelas mesmas letras na coluna não diferem entre si, pelo teste de Tukey, a 5\% de significância.

Tabela 4. Controle de Commelina benghalensis aos 15, 30, 42, 60 e 100 dias após aplicação (DAA) na cultura cafeeira. Viçosa, MG, 2001.

\begin{tabular}{|c|c|c|c|c|c|c|c|}
\hline \multirow[b]{2}{*}{ Tratamento } & \multicolumn{2}{|c|}{ Dose (g/ha) } & \multicolumn{5}{|c|}{ Controle $(\%)^{(1)}$} \\
\hline & $1^{\text {a }}$ Aplicação & $2^{\mathrm{a}}$ Aplicação & $15 \mathrm{DAA}$ & $30 \mathrm{DAA}$ & $42 \mathrm{DAA}$ & $60 \mathrm{DAA}$ & $100 \mathrm{DAA}$ \\
\hline Glyphosate WG & 720 & 1080 & $19 \mathrm{~b}$ & $70 \mathrm{bc}$ & $75 \mathrm{bc}$ & $85 a$ & $83 \mathrm{a}$ \\
\hline Glyphosate WG & 1080 & 1080 & $20 \mathrm{~b}$ & $71 \mathrm{bc}$ & $81 a b c$ & $86 \mathrm{a}$ & $83 \mathrm{a}$ \\
\hline Glyphosate WG & 1440 & 1080 & $40 \mathrm{~b}$ & $85 \mathrm{ab}$ & $92 \mathrm{a}$ & 93 a & $91 \mathrm{a}$ \\
\hline Glyphosate WG & 1800 & 1080 & $30 \mathrm{~b}$ & $80 \mathrm{abc}$ & $88 a b$ & 94 a & $95 \mathrm{a}$ \\
\hline Glyphosate WG & 1080 & --- & $21 \mathrm{~b}$ & $65 c$ & $69 c$ & $53 \mathrm{~b}$ & $51 \mathrm{~b}$ \\
\hline Glyphosate WG & 2160 & --- & $35 \mathrm{~b}$ & $76 \mathrm{abc}$ & $84 a b c$ & $85 \mathrm{a}$ & 84 a \\
\hline Glyphosate SA & 2160 & --- & $34 b$ & $68 \mathrm{bc}$ & $75 b c$ & $80 \mathrm{a}$ & $80 \mathrm{a}$ \\
\hline Glyphosate SA + 2,4-D & $1080+1005$ & --- & $78 \mathrm{a}$ & 93 a & $96 \mathrm{a}$ & 98 a & $96 \mathrm{a}$ \\
\hline Diuron/paraquat & $300+600$ & --- & $75 \mathrm{a}$ & $43 d$ & $21 \mathrm{~d}$ & $9 \mathrm{c}$ & $6 \mathrm{c}$ \\
\hline Testemunha sem capina & --- & --- & 0 & 0 & 0 & 0 & 0 \\
\hline
\end{tabular}

${ }^{(1)}$ Médias seguidas pelas mesmas letras na coluna não diferem entre si, pelo teste de Tukey, a 5\% de significância.

Tabela 5. Toxicidade do cafeeiro aos 15, 30, 42 e 60 dias após aplicação (DAA). Viçosa, MG, 2001.

\begin{tabular}{|c|c|c|c|c|c|c|}
\hline \multirow[b]{2}{*}{ Tratamento } & \multicolumn{2}{|c|}{ Dose (g/ha) } & \multicolumn{4}{|c|}{ Toxicidade $(\%)^{(1)}$} \\
\hline & $1^{2}$ Aplicação & $2^{a}$ Aplicação & $15 \mathrm{DAA}$ & $30 \mathrm{DAA}$ & $42 \mathrm{DAA}$ & $60 \mathrm{DAA}$ \\
\hline Glyphosate WG & 720 & 1080 & $15 b$ & $13 \mathrm{c}$ & $5 b$ & $5 b$ \\
\hline Glyphosate WG & 1080 & 1080 & $20 \mathrm{~b}$ & $14 \mathrm{bc}$ & $8 b$ & $8 \mathrm{~b}$ \\
\hline Glyphosate WG & 1440 & 1080 & $18 \mathrm{~b}$ & $16 \mathrm{bc}$ & $10 \mathrm{~b}$ & $7 b$ \\
\hline Glyphosate WG & 1800 & 1080 & $20 \mathrm{~b}$ & $20 \mathrm{abc}$ & $11 \mathrm{~b}$ & $9 \mathrm{~b}$ \\
\hline Glyphosate WG & 1080 & -- & $21 \mathrm{~b}$ & $11 \mathrm{c}$ & $4 b$ & $3 \mathrm{~b}$ \\
\hline Glyphosate WG & 2160 & --- & $21 \mathrm{~b}$ & $19 \mathrm{abc}$ & $10 \mathrm{~b}$ & $6 \mathrm{~b}$ \\
\hline Glyphosate SA & 2160 & -- & $23 \mathrm{~b}$ & $15 \mathrm{bc}$ & $6 \mathrm{~b}$ & $5 b$ \\
\hline Glyphosate SA + 2,4-D & $1080+1005$ & --- & $41 \mathrm{a}$ & $25 a b$ & $24 \mathrm{a}$ & $25 \mathrm{a}$ \\
\hline Diuron/paraquat & $300 / 600$ & -- & $43 \mathrm{a}$ & $30 \mathrm{a}$ & $29 a$ & $26 \mathrm{a}$ \\
\hline Testemunha sem capina & --- & -- & 0 & 0 & 0 & 0 \\
\hline
\end{tabular}

${ }^{(1)}$ Médias seguidas pelas mesmas letras na coluna não diferem entre si, pelo teste de Tukey, a 5\% de significância. 
A aplicação seqüencial de glyphosate é uma alternativa viável no controle de espécies que normalmente são controladas por produtos que exigem maior cuidado no momento da aplicação.

As formulações glyphosate SA e glyphosate WG, ambas na dose de $2160 \mathrm{~g}$ /ha e.a., não diferiram significativamente quanto à injúria ao cafeeiro. Da mesma forma, não ocorreu diferença entre o parcelamento da dose de glyphosate WG, por meio da aplicação seqüencial, e em única aplicação, quanto às injúrias ocasionadas ao cafeeiro.

\section{CONCLUSÕES}

A mistura em tanque glyphosate $+2,4-\mathrm{D}$ (na dose $1080+1005 \mathrm{~g} / \mathrm{ha}$ e.a.) é eficaz no controle das espécies Commelina diffusa e $C$. benghalensis até os 100 DAA.

A mistura diuron/paraquat (na dose de $300 / 600 \mathrm{~g} /$ ha) não é eficaz controle das espécies $C$. diffusa e C. benghalensis.

A aplicação seqüencial de glyphosate WG, nas doses de 1440 e $1080 \mathrm{~g} /$ ha e.a., com a segunda aplicação 21 dias após a primeira, é eficaz no controle de $C$. diffusa e $C$. benghalensis, até os 100 DAA.

Todos os herbicidas avaliados provocam injúrias ao cafeeiro caso atijam as folhas; entretanto, esta injúria é maior para a mistura em tanque glyphosate $+2,4-\mathrm{D}$ e a mistura formulada diuron/paraquat.

A aplicação seqüencial de glyphosate é uma alterna tiva viável no controle de espécies que normalmente são controladas por produtos que exigem maior cuidado no momento da aplicação, como C. diffusa e C. benghalensis.

\section{LITERATURA CITADA}

BARRETO, R. C. Levantamento das espécies de Commelinaceae R.Br. nativas do Brasil. São Paulo: Universidade de São Paulo, 1997. 490p. (Tese de Doutorado).

BLANCO, H. G; OLIVEIRA, D. A.; PUPO, E. I. H. Período de competição de uma comunidade natural de mato em uma cultura de café em formação. Biológico, v.48, n.1, p.9-20, 1982.

BROMILOW, R. H.; CHAMBERLAIN, K.; EVANS, A. A. Physiocochemical aspects of phloem translocation of herbicide. Weed Science, Champaign, v.38, p.305-314, 1990.

DURIGAN, J. C.; GALLI, A. J. B.; LEITE, G. J. Avaliação da eficiência da mistura de glyphosate e 2,4-D para o controle de plantas daninhas em citros. In: CONGRESSO BRASILEIRO DE HERBICIDAS E PLANTAS DA-
NINHAS, 17 Piracicaba,SP, 1988. Resumos... Piracicaba: SBHPD, 1988. p.303-304.

FRANS, R. E. Measuring plant responses. In: WILKINSON, R. E., ed. Research methods in weed science. Southern Weed Science Society, 1972. p. 28-41.

HARTZLER, B. Which glyphosate product is best? (26/01/ 2001) (http:www.weeds.iastate.edu/mgmt/qtr01-1/ glyphosateformulations.htm.)

HESS, F. D. Mechanism of action of inhibitors of amino acid biosynthesis. In: Herbicide action: an intensive course on the activity, seletivity, behavior, and fate of herbicides in plants and soil. West Lafayette, Indiana: Purdue University, 1994. p.344-365.

KISSMANN, K. G. Plantas infestantes e nocivas. 2 ed. São Paulo: BASF Brasileira S. A., 1997. Tomo 1, 825p.

LORENZI, H. Manual de identificação e controle de plantas daninhas: plantio direto e convencional. 5 ed. Nova Odessa, São Paulo: Editora Plantarum, 2000. p.384.

NJOROGE, J. M. Weeds and weed control in coffee. Experimental Agriculture, v.30, p.421-429, 1994.

RAMOS, H. H.; DURIGAN, J. C. Avaliação da eficiência da mistura pronta de glyphosate $+2,4-\mathrm{D}$ no controle da Commelina virginica L. em citrus. Planta Daninha, v.14, n.1, p.33-41, 1996.

RODRIGUES, B. N.; ALMEIDA, F. S. Guia de herbicidas. Londrina, Paraná: Edição dos autores, 1998. 648p.

SANTOS, I. C.; SILVA, A. A.; FERREIRA, F. A.; MIRANDA, G. V.; PINHEIRO, R. A. N. Eficiência de glyphosate no controle de Commelina benghalensis e Commelina diffusa. Planta Daninha, v.19, n.1, p.135143, 2001.

SANTOS, I. C.; SILVA, A. A.; FERREIRA, F. A.; SANTOS, L. D. T. Eficiência do glyphosate no controle de Commelina spp. In: CONGRESSO BRASILEIRO DA CIÊNCIA DAS PLANTAS DANINHAS, 22. Foz do Iguaçu, PR, 2000. Resumos... Londrina, PR: SBCPD, 2000. p.438.

VARGAS, L.; SILVA, A. A.; BORÉM, A.; REZENDE, S. T.; FERREIRA, F. A.; SEDIYAMA, T. Características da resistência de acordo com o mecanismo de ação herbicida. In: VARGAS, L.; SILVA, A. A.; REZENDE, S. T.; FERREIRA, F. A.; SEDIYAMA, T., eds. Resistência de plantas daninhas a herbicidas. Viçosa: UFV, 1999. p.16-34. 